\title{
Pattern of mental health service use and risk of injury: A longitudinal study
}

\author{
Wenbin Liang*, Tanya Chikritzhs \\ National Drug Research Institute, Curtin University, Perth, Australia; ${ }^{*}$ Corresponding Author: w.liang@curtin.edu.au
}

Received 2 October 2011; revised 18 November 2011; accepted 22 December 2011

\begin{abstract}
Objective: The aim of this study is to investigate the association between mental health treatment patterns and risk of injuries among a Western Australian male birth cohort. Method: A population-based birth-cohort of males born between 1980 and 1984 in Western Australia was followed up using linked health data. Results: Participants with mental health conditions were at an increased risk of injury. Those with a continuous mental health treatment pattern without interruption or window periods had lower risk of injury compared to those with treatment interruption or window periods. The adjusted incidence rate ratios (95\% confidence interval) for injury among participants: 1) without a mental condition; 2) with a previous mental condition; 3 ) with a mental condition in the last four years and without interruption in their mental health treatment; and 4) with a mental condition in the last four years with interruptions in mental health service, were 0.38 (0.35 - 0.40), 0.77 (0.71 - 083), 1.0 (reference group) and $2.06(1.72$ - 2.47) respectively. Conclusion: Increasing resources for mental health services and enabling sufficient continuous mental health services and follow-up may reduce the risk of injury among populations with mental health conditions.
\end{abstract}

Keywords: Injuries; Mental Health Care; Risk; Cohort Study; Australia

\section{INTRODUCTION}

The association between mental illness and risk of intentional and unintentional injuries has been investigated in a number of studies [1-10]. Findings are largely consistent, suggesting that patients with mental conditions are at an increased risk of both intentional and unintentional injuries [1-10]. Mental illness has been shown to be highly prevalent in various cultures and mental health services are often unable to meet treatment needs [11-16]. Moreover, it is possible that risk of injury among populations with mental illness may be influenced by availability and adequacy of mental health services. There is limited data, however, on the potential beneficial effects of mental health service provision on injury prevention. A clearer understanding of the potential link between mental health services and injury among populations with mental illness may enable implementation of evidence-based and potentially cost-effective prevention strategies in the future.

As is the case in many other parts of the world, mental disorders are common among the Australian population [17]. Mental health services in Australia have been improved overtime, but some have argued that limited funding has affected the sector's ability to meet demand [15,17-20]. Continuous service improvement can further reduce the burden of mental disorders, however, it is also important to investigate whether co-morbidities, such as injuries, which are partly due to mental illness, may also benefit. Therefore this study aims to investigate the association between mental health treatment patterns and risk of injuries among a Western Australian male birth cohort.

\section{METHOD}

This study was a population-based birth-cohort study of males born between 1980 and 1984 in Western Australia (WA) using linked health data available through the Western Australian Data Linkage System (WADLS) [21]. Males were identified using birth registry records and observed from age 12 yrs to December 31st, 2009 or death, which ever occurred first. Thus, consecutive years of follow-up ranged from 13 to 17 years. As a typical starting point for adolescence, age 12 yrs was designated the starting point for follow-up [22].

Demographics (year of birth, age of parents, residential location), hospitalizations for physical health conditions (before 12 yrs and during follow-up period) and records of mental health conditions (before 12 yrs and during follow-up period) for the cohort were obtained from a range of sources and linked by the WADLS. Data sources included: 1) Midwives Notifications Data; 2) mental 
health service records from the WA Mental Health Ambulatory Data System; 3) WA Hospital Morbidity Data System; 4) Birth Registry Data; and 5) Mortality Data. Participants who died before the end of follow-up period (Dec 30th 2009) were identified from WA Mortality Data records. The Midwives Notifications Data covered all births attended by a registered midwife (more than $99 \%$ of all births) and the WA Hospital Morbidity Data System included separations from all WA public and private acute hospitals [21]. The reliability and quality of these linked data have been confirmed previously [21].

The WA Mental Health Ambulatory Data System and the WA Hospital Morbidity Data System included all hospitalization records from WA public and private psychiatric hospitals and all attendances at public psychiatric outpatient clinics [21]. In both databases, diagnoses of all mental disorders were coded with either ICD-9 codes (209319) or ICD-10 codes (F00-F99), including for instance, mood disorders, anxiety disorders, substance related disorders, personality disorders and schizophrenia. In this study, mental health care services and treatments were defined as health care services that were provided to treat mental disorders. Mental health services and treatments provided in all WA public and private psychiatric hospitals and at public psychiatric outpatient clinics over the observation period were included in this study. For outpatient services, episode start and end dates were used to define the mental health service interval; for episodes without an end date, the date of the last contact recorded for the same episode was taken as the end date. In the Hospital Morbidity Data, diagnosis was coded using ICD-9 for the period 19791999 and ICD-10 from 1999-2009. Injury diagnosis codes: ICD-9 codes (800 - 995) and ICD-10 codes (S00-S99, T00-T98, V00-V99, W00-W99, X00-X59) were used to identify injury cases that resulted in hospitalization: including but not limited to dislocation, sprain, strain of joints and ligaments; injury of muscle and tendon, superficial injury; open wound, burns and corrosions; fracture.

To control for potential confounding effects of socioeconomic status, residential location (Local Government Area, LGA) at birth was coded according to the Australia Bureau of Statistics' (ABS) Index of Relative Socio-Economic Disadvantage (IRSED) and Index of Education and Occupation (IEO). IRSED is a general measure of economic disadvantage of residential families in an area and the IEO is a general measure of the proportion of residents with higher levels of education and skilled occupations in an area [23].

\subsection{Statistical Analysis}

In this study, the association between mental disorder and injury was assessed for each year of follow-up. Person per unit data was first converted into person-years per unit data. Each person-year unit was defined by unique linked
ID and the age of the person at the time the person-year was observed. Demographic data in the Midwives Notifications Data, mental disorder records from WA Mental Health Ambulatory Data System and WA Hospital Morbidity Data, records of injury hospitalization in the WA Hospital Morbidity Data, and Mortality Data from the WA Mortality Data were extracted linked to its related personyear unit. Multivariate Poisson regression models were employed to estimate incidence rate ratios (IRR).

Mental health service care was measured in two ways. 1) Recent use of mental health service; use of a mental health service in the last four-year-period (separate coding for each year, thus a total of 16 combinations ( 2 to the power of 4). Use of a mental health service was coded separated for each of the past four years for each person-year unit. 2) Previous use of a mental health service: use any mental health service from birth until four years ago was coded as (0 no, 1 yes).

Each model included/controlled for recent use of mental health service, previous use of mental service, calendar year of birth, IRSED and IEO for residential location at birth and age. Analysis was further repeated separately for: 1) 12 - 19 yrs (adolescence phase); and 2) 19 yrs to end of follow-up (adulthood). Post hoc analyses were performed to further investigate the variations of injury risk.

\subsection{Ethics}

Ethical approval for this study was obtained from the Department of Health, Government of Western Australia, Human Research Ethics Committee (DOHWA HREC).

\section{RESULTS AND POST HOC ANALYSIS}

The risk of injury was lowest among participants without records of mental disorders, which was indicated by strata id: 1 (Table 1). It was also observed that the risk of injury varied by different patterns of mental health service use. For example, for both strata 4 and 6 use of mental health services were observed in two out of the last four years, however, for strata 6 the treatment pattern was discontinuous with a "window" period, while for strata 4 , the treatment pattern is continuous. The IRR was higher for strata 6 comparing to strata 4 . Similar differences in relative risks were also observed when comparing treatments without "window" period to those with "window" periods. In Table 2 IRR for treatment patterns shown in Table 1 were displayed based on the orders of the following new categories: 0 , without use of mental health service; 1 , with use of mental health service for only one year in the last four years and/or with use of mental health service 4 years plus ago; 2, with use of mental health service in 2 or more years in the last four years without any window period; 3 , with use of mental service in 2 or more years in the last four years with window period. 
Table 1. Initial multivariate analysis ${ }^{*}$ of risk of injury and mental health treatment patterns.

\begin{tabular}{|c|c|c|c|c|c|c|c|c|c|c|}
\hline \multicolumn{6}{|c|}{ Variables } & Count & \multirow[t]{2}{*}{$\begin{array}{l}\text { Person-year } \\
\text { at risk }\end{array}$} & \multirow[t]{2}{*}{ IRR } & \\
\hline Strata indicators & 4 yrs+ ago & 3 - 4 yrs ago & 2 - 3 yrs ago & $1-2$ yrs ago & $0-1$ yr ago & & & & \multicolumn{2}{|c|}{$\begin{array}{l}95 \% \text { confidence } \\
\text { interval }\end{array}$} \\
\hline 1 & $0^{\wedge}$ & 0 & 0 & 0 & 0 & 17,832 & 763,146 & 0.63 & 0.59 & 0.66 \\
\hline 2 & 0 & 0 & 0 & 0 & 1 & 380 & 3878 & 2.61 & 2.33 & 2.93 \\
\hline 3 & 0 & 0 & 0 & 1 & 0 & 142 & 2159 & 1.75 & 1.47 & 2.08 \\
\hline 4 & 0 & 0 & 0 & 1 & 1 & 154 & 1895 & 2.18 & 1.85 & 2.58 \\
\hline 5 & 0 & 0 & $1^{\wedge}$ & 0 & 0 & 126 & 1958 & 1.71 & 1.43 & 2.06 \\
\hline 6 & 0 & 0 & 1 & 0 & 1 & 18 & 145 & 3.25 & 2.04 & 5.17 \\
\hline 7 & 0 & 0 & 1 & 1 & 0 & 41 & 794 & 1.40 & 1.02 & 1.91 \\
\hline 8 & 0 & 0 & 1 & 1 & 1 & 81 & 1260 & 1.72 & 1.37 & 2.15 \\
\hline 9 & 0 & 1 & 0 & 0 & 0 & 97 & 1854 & 1.40 & 1.14 & 1.72 \\
\hline 10 & 0 & 1 & 0 & 0 & 1 & 8 & 85 & 2.51 & 1.25 & 5.02 \\
\hline 11 & 0 & 1 & 0 & 1 & 0 & 17 & 70 & 6.32 & 3.92 & 10.20 \\
\hline 12 & 0 & 1 & 0 & 1 & 1 & 9 & 71 & 3.34 & 1.73 & 6.43 \\
\hline 13 & 0 & 1 & 1 & 0 & 0 & 35 & 746 & 1.27 & 0.91 & 1.78 \\
\hline 14 & 0 & 1 & 1 & 0 & 1 & 13 & 72 & 4.87 & 2.82 & 8.41 \\
\hline 15 & 0 & 1 & 1 & 1 & 0 & 21 & 404 & 1.40 & 0.91 & 2.16 \\
\hline 16 & 0 & 1 & 1 & 1 & 1 & 57 & 950 & 1.61 & 1.23 & 2.09 \\
\hline 17 & 1 & 0 & 0 & 0 & 0 & 1269 & 34,481 & 1.0 & Refer & \\
\hline 18 & 1 & 0 & 0 & 0 & 1 & 107 & 660 & 4.36 & 3.58 & 5.32 \\
\hline 19 & 1 & 0 & 0 & 1 & 0 & 28 & 442 & 1.70 & 1.17 & 2.47 \\
\hline 20 & 1 & 0 & 0 & 1 & 1 & 55 & 382 & 3.88 & 2.96 & 5.08 \\
\hline 21 & 1 & 0 & 1 & 0 & 0 & 36 & 477 & 2.01 & 1.44 & 2.80 \\
\hline 22 & 1 & 0 & 1 & 0 & 1 & 6 & 69 & 2.31 & 1.04 & 5.15 \\
\hline 23 & 1 & 0 & 1 & 1 & 0 & 14 & 201 & 1.88 & 1.11 & 3.19 \\
\hline 24 & 1 & 0 & 1 & 1 & 1 & 57 & 364 & 4.19 & 3.21 & 5.47 \\
\hline 25 & 1 & 1 & 0 & 0 & 0 & 116 & 2569 & 1.22 & 1.01 & 1.48 \\
\hline 26 & 1 & 1 & 0 & 0 & 1 & 12 & 131 & 2.47 & 1.40 & 4.37 \\
\hline 27 & 1 & 1 & 0 & 1 & 0 & 10 & 105 & 2.54 & 1.36 & 4.72 \\
\hline 28 & 1 & 1 & 0 & 1 & 1 & 23 & 149 & 4.10 & 2.71 & 6.19 \\
\hline 29 & 1 & 1 & 1 & 0 & 0 & 63 & 1586 & 1.07 & 0.83 & 1.38 \\
\hline 30 & 1 & 1 & 1 & 0 & 1 & 17 & 132 & 3.41 & 2.11 & 5.50 \\
\hline 31 & 1 & 1 & 1 & 1 & 0 & 49 & 1160 & 1.14 & 0.86 & 1.51 \\
\hline 32 & 1 & 1 & 1 & 1 & 1 & 269 & 4698 & 1.54 & 1.35 & 1.75 \\
\hline \multicolumn{11}{|l|}{ Birth year } \\
\hline 1980 & & & & & & 4180 & 170,146 & 1.0 & Refer & \\
\hline 1981 & & & & & & 4445 & 177,538 & 1.02 & 0.98 & 1.06 \\
\hline 1982 & & & & & & 4236 & 164,714 & 1.05 & 1.00 & 1.09 \\
\hline 1983 & & & & & & 4426 & 163,624 & 1.10 & 1.06 & 1.15 \\
\hline 1984 & & & & & & 3875 & 151,071 & 1.04 & 1.00 & 1.09 \\
\hline
\end{tabular}




\section{Continued}

\begin{tabular}{|c|c|c|c|c|c|}
\hline \multicolumn{6}{|c|}{ Index of Education and Occupation } \\
\hline $1^{\text {st }}$ quintile & 4692 & 168,214 & 1.0 & \multicolumn{2}{|c|}{ Reference } \\
\hline $2^{\text {nd }}$ quintile & 4645 & 164,599 & 1.09 & 1.04 & 1.13 \\
\hline $3^{\text {rd }}$ quintile & 5280 & 201,836 & 1.07 & 1.02 & 1.13 \\
\hline $4^{\text {th }}$ quintile & 3576 & 155,037 & 0.97 & 0.91 & 1.03 \\
\hline $5^{\text {th }}$ quintile & 2969 & 137,407 & 0.91 & 0.85 & 0.98 \\
\hline \multicolumn{6}{|c|}{ Index of Relative Socio-Economic Disadvantage } \\
\hline $1^{\text {st }}$ quintile & 5284 & 171,443 & 1.0 & \multicolumn{2}{|c|}{ Reference } \\
\hline $2^{\text {nd }}$ quintile & 4054 & 157,447 & 0.84 & 0.80 & 0.88 \\
\hline $3^{\text {rd }}$ quintile & 4605 & 184,827 & 0.80 & 0.76 & 0.85 \\
\hline $4^{\text {th }}$ quintile & 4596 & 190,135 & 0.78 & 0.74 & 0.82 \\
\hline $5^{\text {th }}$ quintile & 2623 & 123,241 & 0.77 & 0.72 & 0.83 \\
\hline \multicolumn{6}{|c|}{ Observation Period } \\
\hline $12-18$ & 9118 & 364,599 & 1.0 & \multicolumn{2}{|c|}{ Reference } \\
\hline $19+$ & 12,044 & 462,494 & 1.02 & 0.99 & 1.04 \\
\hline
\end{tabular}

${ }^{\wedge}$ Zero and without filling indicates without use of mental service. One and with filling indicates with mental service; ${ }^{*}$ Adjusted for age, calendar year of birth, IEO and IRSED.

Table 2. Mental health treatment patterns explain part of risk variation (within the new strata groups, old strata were sorted by the size of the IRR). ${ }^{*}$

\begin{tabular}{|c|c|c|c|c|c|c|c|c|c|}
\hline $\begin{array}{l}\text { New strata } \\
\text { indicators }\end{array}$ & $\begin{array}{c}\text { Strata } \\
\text { indicators }\end{array}$ & 4 yrs+ ago & 3 - 4 yrs ago & 2 - 3 yrs ago & 1 - 2 yrs ago & 0 - 1 yr ago & IRR & \multicolumn{2}{|c|}{$\begin{array}{l}\text { 95\% confidence } \\
\text { interval }\end{array}$} \\
\hline 0 & 1 & $0^{\wedge} \wedge$ & 0 & 0 & 0 & 0 & 0.63 & 0.59 & 0.66 \\
\hline 1 & 17 & 1 & 0 & 0 & 0 & 0 & 1.0 & \multicolumn{2}{|c|}{ Reference } \\
\hline 1 & 25 & 1 & 1 & 0 & 0 & 0 & 1.22 & 1.01 & 1.48 \\
\hline 1 & 9 & 0 & 1 & 0 & 0 & 0 & 1.4 & 1.14 & 1.72 \\
\hline 1 & 19 & 1 & 0 & 0 & 1 & 0 & 1.7 & 1.17 & 2.47 \\
\hline 1 & 5 & 0 & 0 & 1 & 0 & 0 & 1.71 & 1.43 & 2.06 \\
\hline 1 & 3 & 0 & 0 & 0 & 1 & 0 & 1.75 & 1.47 & 2.08 \\
\hline 1 & 21 & 1 & 0 & 1 & 0 & 0 & 2.01 & 1.44 & 2.8 \\
\hline 1 & 2 & 0 & 0 & 0 & 0 & $1^{\wedge}$ & 2.61 & 2.33 & 2.93 \\
\hline 1 & 18 & 1 & 0 & 0 & 0 & 1 & 4.36 & 3.58 & 5.32 \\
\hline 2 & 29 & 1 & 1 & 1 & 0 & 0 & 1.07 & 0.83 & 1.38 \\
\hline 2 & 31 & 1 & 1 & 1 & 1 & 0 & 1.14 & 0.86 & 1.51 \\
\hline 2 & 13 & 0 & 1 & 1 & 0 & 0 & 1.27 & 0.91 & 1.78 \\
\hline 2 & 7 & 0 & 0 & 1 & 1 & 0 & 1.4 & 1.02 & 1.91 \\
\hline 2 & 15 & 0 & 1 & 1 & 1 & 0 & 1.4 & 0.91 & 2.16 \\
\hline 2 & 32 & 1 & 1 & 1 & 1 & 1 & 1.54 & 1.35 & 1.75 \\
\hline 2 & 16 & 0 & 1 & 1 & 1 & 1 & 1.61 & 1.23 & 2.09 \\
\hline 2 & 8 & 0 & 0 & 1 & 1 & 1 & 1.72 & 1.37 & 2.15 \\
\hline 2 & 23 & 1 & 0 & 1 & 1 & 0 & 1.88 & 1.11 & 3.19 \\
\hline 2 & 4 & 0 & 0 & 0 & 1 & 1 & 2.18 & 1.85 & 2.58 \\
\hline 2 & 20 & 1 & 0 & 0 & 1 & 1 & 3.88 & 2.96 & 5.08 \\
\hline 2 & 24 & 1 & 0 & 1 & 1 & 1 & 4.19 & 3.21 & 5.47 \\
\hline 3 & 22 & 1 & 0 & 1 & 0 & 1 & 2.31 & 1.04 & 5.15 \\
\hline 3 & 26 & 1 & 1 & 0 & 0 & 1 & 2.47 & 1.4 & 4.37 \\
\hline 3 & 10 & 0 & 1 & 0 & 0 & 1 & 2.51 & 1.25 & 5.02 \\
\hline 3 & 27 & 1 & 1 & 0 & 1 & 0 & 2.54 & 1.36 & 4.72 \\
\hline 3 & 6 & 0 & 0 & 1 & 0 & 1 & 3.25 & 2.04 & 5.17 \\
\hline 3 & 12 & 0 & 1 & 0 & 1 & 1 & 3.34 & 1.73 & 6.43 \\
\hline 3 & 30 & 1 & 1 & 1 & 0 & 1 & 3.41 & 2.11 & 5.5 \\
\hline 3 & 28 & 1 & 1 & 0 & 1 & 1 & 4.1 & 2.71 & 6.19 \\
\hline 3 & 14 & 0 & 1 & 1 & 0 & 1 & 4.87 & 2.82 & 8.41 \\
\hline 3 & 11 & 0 & 1 & 0 & 1 & 0 & 6.32 & 3.92 & 10.2 \\
\hline
\end{tabular}

${ }^{\wedge}$ Zero and without filling indicates without use of mental service. One and with filling indicates with mental service; ${ }^{*}$ Adjusted for age, calendar year of birth, IEO and IRSED. 
The IRR for group 3 were generally higher than those in groups 2 and 1 (Table 2). Therefore a post hoc analysis was performed to compare the three new combined groups. Multivariate Poisson regression model was used adjusting for all variables as the initial Poisson model. Analysis was further repeated for periods of 12 - 18 yrs and 19 yrs+ separately. Results of post-hoc analysis are shown in Table 3. Mental health service with window periods was associated with increased risk comparing to mental health service without window periods when analyses were performed for the whole follow-up period as well as for separated periods 12 - 18 yrs and 19 yrs+.

\section{DISCUSSION}

In this study, the association between use of mental health service and risk of injury were examined in a population-based male cohort study. Consistent with findings from previous studies [1-10], it was observed that participants with mental health conditions and who required mental health services were at an increased risk of injury. It was also observed that continuous mental health treatment pattern without interruption or window periods was associated with lower risk of injury compared to those with treatment interruption or window periods. To the best of our knowledge, there is no published data on mental health treatment patterns in relations to overall injury risk.
As discussed above, mental disorders can be risk factors for both intentional and unintentional injuries, and thus effective treatment of mental illness may reduce the risk of injuries. In Australia, as well as many other countries, the gap between demand and supply of mental health services remained, though some improvement has occurred in the last few years [15,17-20]. Inadequacy of mental health services may also be one of the causes of hospitalizations for injury, providing sufficient funding to fully meet the demands of mental health services may therefore further reduce the burden of injury on the health care sector while promoting mental health. Nevertheless some patients with mental disorders may miss follow-up appointments and drop out of treatments frequently. Innovative programs and resources to improve follow-up as well as to provide additional supports are required to reduce the risk of injury among patients with mental conditions [24-26].

\section{Limitations}

The main limitation of this study is that it only includes males so the association observed should be further tested in a population-based female cohort. In this study, we excluded records of intentional injuries from the analysis; however, misclassifications between intentional injuries and unintentional injuries have been common in clinical practice. Therefore we could not assume that all intentional injuries were excluded.

Table 3. Post hoc multivariate analysis* of risk of injury and types of mental health treatment patterns.

\begin{tabular}{|c|c|c|c|c|c|}
\hline & Count & Person-years & IRR & \multicolumn{2}{|c|}{ 95\% confidence interval } \\
\hline \multicolumn{6}{|c|}{12 yrs $^{+}$} \\
\hline 0 & 17,832 & 763,146 & 0.38 & 0.35 & 0.40 \\
\hline 1 & 2301 & 48,478 & 0.77 & 0.71 & 0.83 \\
\hline 2 & 896 & 14,440 & 1.00 & \multicolumn{2}{|c|}{ Reference } \\
\hline 3 & 133 & 1029 & 2.06 & 1.72 & 2.47 \\
\hline \multicolumn{6}{|c|}{$12-18$ yrs } \\
\hline 0 & 8181 & 344,294 & 0.51 & 0.45 & 0.57 \\
\hline 1 & 592 & 13,079 & 0.95 & 0.83 & 1.08 \\
\hline 2 & 321 & 6960 & 1.00 & \multicolumn{2}{|c|}{ Reference } \\
\hline 3 & 24 & 266 & 1.85 & 1.22 & 2.80 \\
\hline \multicolumn{6}{|c|}{19 yrs $+^{+}$} \\
\hline 0 & 9651 & 418,852 & 0.30 & 0.28 & 0.33 \\
\hline 1 & 1709 & 35,399 & 0.65 & 0.59 & 0.72 \\
\hline 2 & 575 & 7480 & 1.00 & \multicolumn{2}{|c|}{ Reference } \\
\hline 3 & 109 & 763 & 1.89 & 1.54 & 2.31 \\
\hline
\end{tabular}

*Adjusted for age, calendar year of birth, IEO and IRSED. 


\section{CONCLUSION}

In this study, it was observed that mental health treatment patterns interrupted with window periods was associated was increased risk of injury compared to continuous treatment patterns. Increasing resources for mental health services and enabling sufficient continuous mental health services may reduce the risk of injury among populations with mental conditions.

\section{REFERENCES}

[1] Brehaut, J.C., Miller, A., Raina, P. and McGrail, K.M. (2003) Childhood behavior disorders and injuries among children and youth: A population-based study. Pediatrics, 111, 262-269. doi:10.1542/peds.111.2.262

[2] Cameron, C., Purdie, D., Kliewer, E. and McClure, R. (2006) Mental health: A cause or consequence of injury? A population-based matched cohort study. BMC Public Health, 6, 114. doi:10.1186/1471-2458-6-114

[3] DiScala, C., Lescohier, I., Barthel, M. and Li, G. (1998) Injuries to children with attention deficit hyperactivity disorder. Pediatrics, 102, 1415-1421. doi:10.1542/peds.102.6.1415

[4] Jokela, M., Ferrie, J. and Kivimäki, M. (2009) Childhood problem behaviors and death by midlife: The british national child development study. Journal of the American Academy of Child \& Adolescent Psychiatry, 48, 19-24. doi:10.1097/CHI.0b013e31818b1c76

[5] Jokela, M., Power, C. and Kivimäki, M. (2009) Childhood problem behaviors and injury risk over the life course. Journal of Child Psychology and Psychiatry, 50, 1541-1549. doi:10.1111/j.1469-7610.2009.02122.x

[6] Neeleman, J., Wessely, S. and Wadsworth, M. (1998) Predictors of suicide, accidental death, and premature natural death in a general-population birth cohort. The Lancet, 351, 93-97. doi:10.1016/S0140-6736(97)06364-2

[7] Rowe, R., Maughan, B. and Goodman, R. (2004) Childhood psychiatric disorder and unintentional injury: Findings from a national cohort study. Journal of Pediatric Psychology, 29, 119-130. doi:10.1093/jpepsy/jsh015

[8] Schwebel, D.C., Speltz, M.L., Jones, K. and Bardina, P. (2002) Unintentional injury in preschool boys with and without early onset of disruptive behavior. Journal of Pediatric Psychology, 27, 727-737.

doi:10.1093/jpepsy/27.8.727

[9] Simon, G.E. and Savarino, J. (2007) Suicide attempts among patients starting depression treatment with medications or psychotherapy. American Journal of Psychiatry, 164, 1029-1034. doi:10.1176/appi.ajp.164.7.1029

[10] Wan, J.J., Morabito, D.J., Khaw, L., Knudson, M.M. and Dicker, R.A. (2006) Mental illness as an independent risk factor for unintentional injury and injury recidivism. The Journal of Trauma, 61, 1299-1304. doi:10.1097/01.ta.0000240460.35245.1a

[11] Wang, P.S., Aguilar-Gaxiola, S., Alonso, J., Angermeyer, M.C., Borges, G., Bromet, E.J., et al. (2007) Use of men- tal health services for anxiety, mood, and substance disorders in 17 countries in the WHO world mental health surveys. The Lancet, 370, 841-850. doi:10.1016/S0140-6736(07)61414-7

[12] Ustun, T.B., Ayuso-Mateos, J.L., Chatterji, S., Mathers, C. and Murray, C.J.L. (2004) Global burden of depressive disorders in the year 2000. The British Journal of Psychiatry, 184, 386-392. doi:10.1192/bjp.184.5.386

[13] Bijl, R.V., Ravelli, A. and van Zessen, G. (1998) Prevalence of psychiatric disorder in the general population: Results of the Netherlands Mental Health Survey and Incidence Study (NEMESIS). Social Psychiatry and Psychiatric Epidemiology, 33, 587-595. doi:10.1007/s001270050098

[14] Wittchen, H.-U. and Jacobi, F. (2005) Size and burden of mental disorders in Europe-A critical review and appraisal of 27 studies. European Neuropsychopharmacology, 15, 357-376. doi:10.1016/j.euroneuro.2005.04.012

[15] Teesson, M., Slade, T. and Mills, K. (2009) Comorbidity in Australia: Findings of the 2007 national survey of mental health and wellbeing. Australian and New Zealand Journal of Psychiatry, 43, 606-614. doi:10.1080/00048670902970908

[16] Wang, P.S., Lane, M., Olfson, M., Pincus, H.A., Wells, K.B. and Kessler, R.C. (2005) Twelve-month use of mental health services in the United States: Results from the national comorbidity survey replication. Archives of General Psychiatry, 62, 629-640. doi:10.1001/archpsyc.62.6.629

[17] Slade, T., Johnston, A., Oakley Browne, M.A., Andrews, G. and Whiteford, H. (2009) 2007 National survey of mental health and wellbeing: Methods and key findings. Australian and New Zealand Journal of Psychiatry, 43, 594-605. doi:10.1080/00048670902970882

[18] Council of Australian Governments (2008) Towards recovery: Mental health services in Australia. Commonwealth of Australia, Canberra.

[19] DOHA, National Mental Health Report 2007: Summary of twelve years of reform in Australia's mental health services under the National Mental Health Strategy 1993-2005. 2007, Commonwealth of Australia, Canberra.

[20] Burgess, P.M., Pirkis, J.E., Slade, T.N., Johnston, A.K., Meadows, G.N. and Gunn, J.M. (2009) Service use for mental health problems: Findings from the 2007 National Survey of Mental Health and Wellbeing. Australian and New Zealand Journal of Psychiatry, 43, 615-623. doi:10.1080/00048670902970858

[21] Holman, C.D.J., Bass, A.J., Rouse, I.L. and Hobbs, M.S.T. (1999) Population-based linkage of health records in Western Australia: Development of a health services research linked database. Australian and New Zealand Journal of Public Health, 23, 453-459. doi:10.1111/j.1467-842X.1999.tb01297.x

[22] Hales, R.E., Yudofsky, S.C. and Gabbard, G.O. (2010) The American psychiatric publishing textbook of psychiatry. 5th Edition, American Psychiatric Publishing, Washington DC.

[23] McLennan, W. (1998) 1996 census of population and 
housing: Socio-economic indexes for areas, A.B.o. Statistics. Australian Bureau of Statistics, Canberra.

[24] Bateman, A.W. and Fonagy, P. (2000) Effectiveness of psychotherapeutic treatment of personality disorder. The British Journal of Psychiatry, 177, 138-143. doi:10.1192/bjp.177.2.138

[25] Carter, G.L., Clover, K., Whyte, I.M., Dawson, A.H. and Este, C.D. (2005) Postcards from the EDge project: Ran- domised controlled trial of an intervention using postcards to reduce repetition of hospital treated deliberate self poisoning. $B M J, \mathbf{3 3 1}, 805$. doi:10.1136/bmj.38579.455266.E0

[26] Mitchell, A.J. and Selmes, T. (2007) Why don’t patients attend their appointments? Maintaining engagement with psychiatric services. Advances in Psychiatric Treatment, 13, 423-434. doi:10.1192/apt.bp.106.003202 